CRYSTALLOGRAPHIC COMMUNICATIONS

ISSN 2056-9890

Received 13 June 2017

Accepted 3 July 2017

Edited by A. V. Yatsenko, Moscow State University, Russia

Keywords: crystal structure; benzimidazole; hydrogen bonding; Hirshfeld surface analysis; DFT calculations; UV spectra.

CCDC reference: 1548944

Supporting information: this article has supporting information at journals.iucr.org/e


OPEN $\odot$ ACCESS

\section{Crystal structure and UV spectra of a 1,2-disubstituted benzimidazolium chloride}

Tuhin Khan, ${ }^{*}$ Navneet Mishra, Darshan S. Mhatre and Anindya Datta

Department of Chemistry, Indian Institute of Technology Bombay, Mumbai 400 076, India. *Correspondence e-mail: tuhin@chem.iitb.ac.in

1-(2-Hydroxybenzyl)-2-(2-hydroxyphenyl)-1H-benzimidazol-3-ium chloride, $\mathrm{C}_{20} \mathrm{H}_{17} \mathrm{~N}_{2} \mathrm{O}_{2}{ }^{+} \cdot \mathrm{Cl}^{-}$, was prepared by reaction of salicylaldehyde with $o$-phenylenediamine in the presence of trimethylsilyl chloride acting as a source of $\mathrm{HCl}$. As a result of steric hindrance, the cation in the crystal is far from planar: the benzimidazole ring system makes dihedral angles of $55.49(9)$ and $81.36(8)^{\circ}$ with the planes of the phenolic groups. The crystal packing is dominated by $\mathrm{O}-$ $\mathrm{H} \cdots \mathrm{Cl}$ and $\mathrm{N}-\mathrm{H} \cdots \mathrm{Cl}$ hydrogen bonds, which link the cations and anions into four-membered rings and then into chains along [100]. The title compound exhibits two transitions in the UV region, which are revealed in the solid state and solution spectra as an absorption maximum at $280 \mathrm{~nm}$ and a shoulder at $320 \mathrm{~nm}$. According to the results of TD-DFT calculation, both transitions have a $\pi-\pi^{*}$ nature and the molecular orbitals involved in these transitions are mostly localized on the benzimidazole ring system and on the phenyl ring attached to it at the 2-position.

\section{Chemical context}

Benzimidazole derivatives are well known to exhibit antibacterial, antimalarial and anti-inflammatory properties (Keri et al., 2015; Carvalho et al., 2011). Besides this, 1,2-disubstituted benzimidazoles are used as intermediates in synthesis of dyes and pigments (Carvalho et al., 2011). Some substituted benzimidazoles, e.g. 2-(2'-hydroxyphenyl)benzimidazole and its derivatives, are strongly fluorescent and show dual emission due to the excited state proton transfer (Douhal et al., 1994). In the solid state, these compounds exhibit fluorescence, which is governed by their polymorphism and steric effects (Konoshima et al., 2012; Benelhadj et al., 2013; Shida et al., 2013). Thus, this class of compounds is considered for applications in fluorescence imaging and optoelectronics (Zhao et al., 2011). Benzimidazolium salts attract attention due to their non-linear optical properties (Sun et al., 2011; Wang et al., 2011). 2-(2'-Hydroxyphenyl)benzimidazole, which is a member of this class of compounds, exhibits rotamerism (Ríos Vazquez et al., 2008). In this work, the crystal structure of 1-(2hydroxybenzyl)-2-(2-hydroxyphenyl)-1H-benzimidazol-3-ium chloride and its UV spectra have been reported. DFT calculations were carried out to study the geometry and electronic transitions.

\section{Structural commentary}

All bond lengths and bond angles are within the ranges reported for similar structures (Ha, 2012). The asymmetric unit, consisting of a 1-(2-hydroxybenzyl)-2-(2-hydroxyphen- 
yl)-1H-benzo[ $d]$ imidazol-3-ium cation and a chloride anion, is presented in Fig. 1. As a result of steric hindrance, the cation is far from planar: the benzimidazole ring system makes dihedral angles of $55.49(9)$ and $81.36(8)^{\circ}$ with the planes of phenolic groups immediately attached to it at position 2 and linked via the methylene bridge to position 1 , respectively. The deviation from planarity in the 2-(2-hydroxyphenyl)benzimidazolium skeleton is larger than in the reported similar structures (AlDouh et al., 2009b; Wang et al., 2011).<smiles>Oc1ccccc1Cn1c(-c2ccccc2O)[nH+]c2ccccc21</smiles>

\section{Supramolecular features}

In the crystal, each cation forms three hydrogen bonds, two $\mathrm{O}-\mathrm{H} \cdots \mathrm{Cl}$ and one $\mathrm{N}-\mathrm{H} \cdots \mathrm{Cl}$ (Table 1), to chloride anions. As a result of these interactions, the cations and anions form ribbons along [100], which consist of centrosymmetric four-

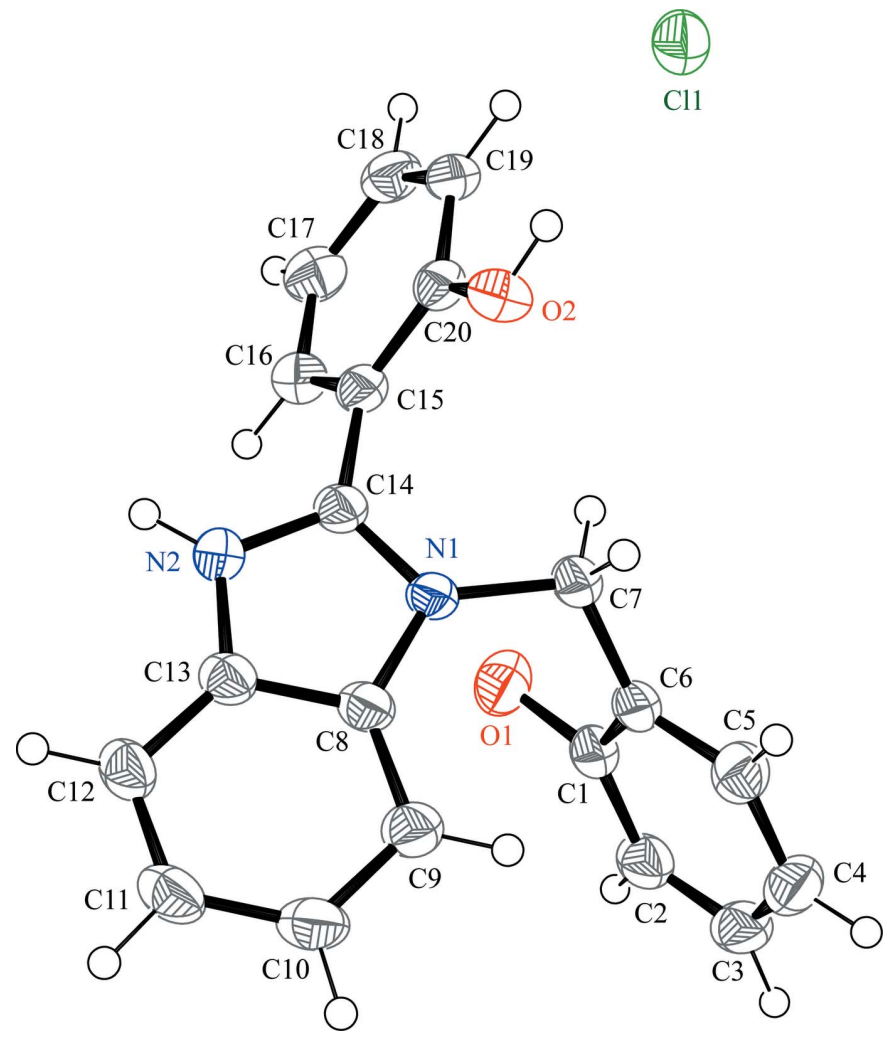

Figure 1

ORTEP diagram of the title compound with displacement ellipsoids drawn at the $50 \%$ probability level.
Table 1

Hydrogen-bond geometry $\left(\AA,{ }^{\circ}\right)$.

\begin{tabular}{lllll}
\hline$D-\mathrm{H} \cdots A$ & $D-\mathrm{H}$ & $\mathrm{H} \cdots A$ & $D \cdots A$ & $D-\mathrm{H} \cdots A$ \\
\hline $\mathrm{O} 1-\mathrm{H} 1 \cdots \mathrm{Cl} 1^{\mathrm{i}}$ & 0.84 & 2.24 & $3.066(2)$ & 169 \\
$\mathrm{O} 2-\mathrm{H} 2 \cdots \mathrm{Cl} 1$ & 0.84 & 2.23 & $3.071(1)$ & 177 \\
$\mathrm{~N} 2-\mathrm{H} 2 A \cdots \mathrm{Cl} 1^{\mathrm{ii}}$ & 0.88 & 2.23 & $3.084(2)$ & 162 \\
$\mathrm{C} 16-\mathrm{H} 16 \cdots \mathrm{O} 1^{\text {iii }}$ & 0.95 & 2.57 & $3.253(2)$ & 129 \\
$\mathrm{C} 19-\mathrm{H} 19 \cdots \mathrm{Cl} 1$ & 0.95 & 2.93 & $3.646(2)$ & 134 \\
$\mathrm{C} 7-\mathrm{H} 7 B \cdots C g(\mathrm{C} 1-\mathrm{C} 6)^{\text {iv }}$ & 0.99 & 2.77 & $3.500(2)$ & 131
\end{tabular}

Symmetry codes: (i) $x+1, y, z$; (ii) $-x,-y+1,-z+1$; (iii) $-x+1,-y+1,-z+1$; (iv) $-x+1,-y+1,-z+2$.

membered rings each formed by two cations and two anions in the $R_{4}^{2}(16)$ and $R_{4}^{2}(20)$ manner, as shown in Fig. 2. Some weak contacts $\mathrm{C}-\mathrm{H} \cdots \mathrm{O}, \mathrm{C}-\mathrm{H} \cdots \mathrm{Cl}$ and $\mathrm{C}-\mathrm{H} \cdots \pi$ are also present (Table 1).

\section{Hirshfeld surface analysis}

To evaluate the effect of close range interactions and compare their significance, Hirshfeld surface analysis (Spackman \& Jayatilaka, 2009; Soman et al., 2014) has been performed and its results are presented in Fig. 3. Four red spots on the Hirshfeld surface indicate short contacts. All close interactions are mediated by $\mathrm{Cl}^{-}$anions. The $\mathrm{H} \cdots \mathrm{H}$ and $\mathrm{C} \cdot \mathrm{H}$ interactions are associated with $46 \%$ and $26 \%$ surface area, respectively. The contributions of the $\mathrm{Cl} \cdots \mathrm{H}(15 \%)$ and $\mathrm{O} \cdots \mathrm{H}(6 \%)$ interactions are smaller, but significant for the crystal architecture.

\section{Quantum chemical calculation}

The geometry of the cation-anion pair in the gas phase was optimized with density functional theory (DFT) using GAUSSIAN09 package (Frisch et al., 2009) within the framework of B3LYP/6-31G(d). Frequency calculations were carried out to confirm that the structure corresponds to a minimum. The optimized bond lengths agree with those

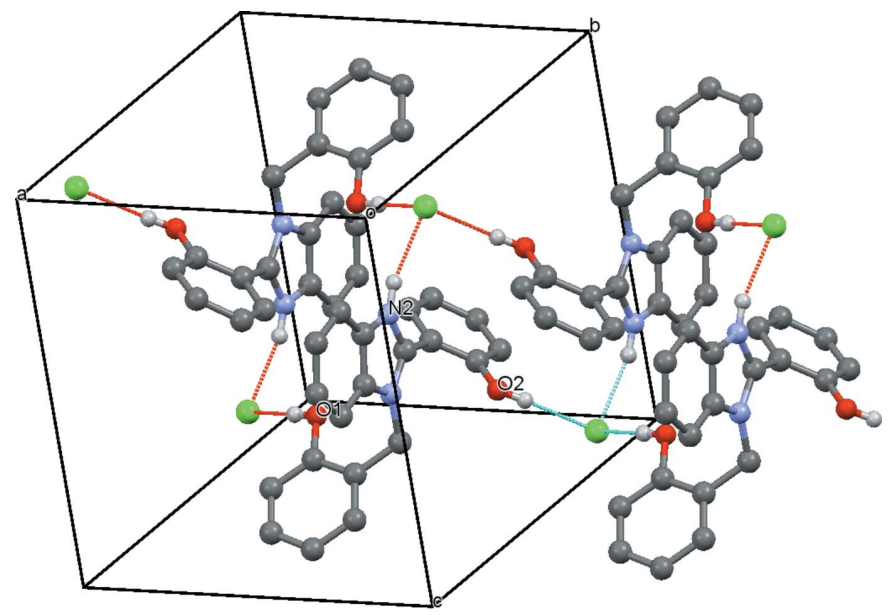

Figure 2

Packing diagram highlighting the hydrogen-bonding interactions. 
Table 2

Comparison of notable bond lengths and torsion angles $\left(\AA{ }^{\circ}\right)$.

\begin{tabular}{lll}
\hline & Crystal & DFT optimized \\
\hline $\mathrm{C} 20-\mathrm{O} 2$ & $1.356(2)$ & 1.315 \\
$\mathrm{C} 20-\mathrm{C} 19$ & $1.391(3)$ & 1.413 \\
$\mathrm{C} 14-\mathrm{N} 2$ & $1.337(2)$ & 1.356 \\
$\mathrm{C} 14-\mathrm{N} 1$ & $1.344(2)$ & 1.349 \\
$\angle \mathrm{C} 16-\mathrm{C} 15-\mathrm{C} 14-\mathrm{N} 1$ & $123.4(2)$ & 137.99 \\
$\angle \mathrm{C} 20-\mathrm{C} 15-\mathrm{C} 14-\mathrm{N} 2$ & $125.5(2)$ & 132.45 \\
\hline
\end{tabular}

observed in the crystal structure within the range of $0.04 \AA$ (Table 2). The largest distinction between the calculated and crystallographic geometries is related to the twist of the phenolic group attached to the benzimidazole ring system at position 2: in the crystal, the corresponding torsion angles are by $7-14^{\circ}$ nearer to $180^{\circ}$ than the calculated values (Table 2 ). This could be due to the hydrogen-bonding and $\mathrm{C}-\mathrm{H} \cdots \pi$ interactions. The ionic nature of the optimized cation-anion pair is reflected in the large calculated dipole moment of 18.05 D. The time-dependent DFT (TD-DFT) calculation was performed on the crystal geometry at the same level of theory as for geometry optimization.

\section{UV spectra}

The solid-state diffuse reflectance spectrum was measured with a Shimadzu-3600 spectrophotometer fitted with an MPC3100 sample compartment. For that, the crystals were crushed to powder and mixed with $\mathrm{BaSO}_{4}$ to a final concentration of $5 \%(v / v)$. The Kubelka-Munk transformation (Kubelka \& Munk, 1931) was applied to the reflectance data. The spectrum of methanol solution was measured with JASCO V530 spectrophotometer. The solid-state spectrum closely resembles the spectrum of the solution, thus indicating that the geometry and electronic structure of the cation did not change in moving from solid state to solution. In the UV region, the title

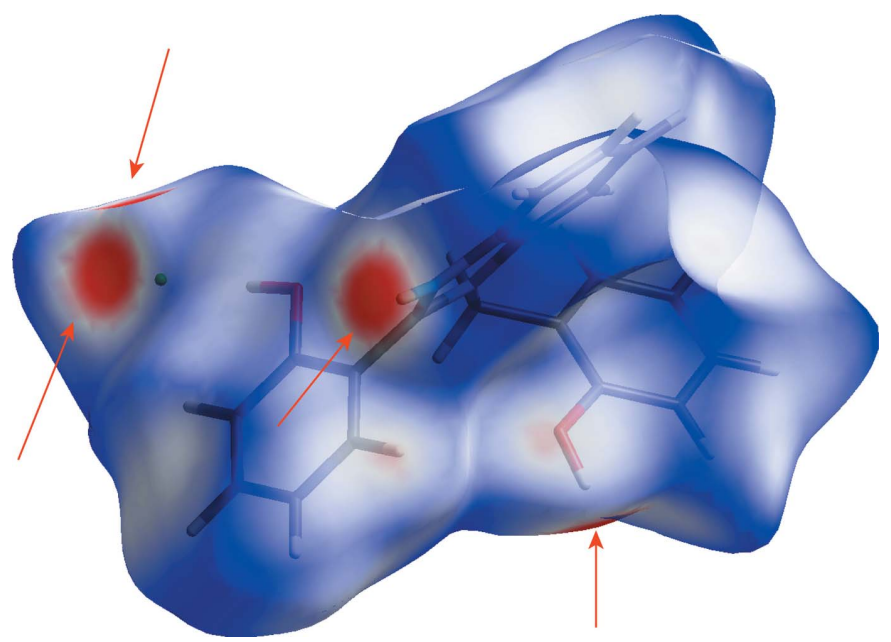

Figure 3

Hirshfeld surface of the ionic pair mapped with normalized contact distances $\left(d_{\text {norm }}\right)$ indicated by red spots. Positions of close contacts are highlighted by red arrows.
Table 3

Prominent electronic transitions obtained from TD-DFT calculation.

\begin{tabular}{lll}
\hline Wavelength & Oscillator strength & Transition \\
\hline $356 \mathrm{~nm}$ & 0.088 & LUMO $\leftarrow$ HOMO-3 $(98 \%)$ \\
$277 \mathrm{~nm}$ & 0.2827 & LUMO $\leftarrow$ HOMO-5 $(96 \%)$ \\
$253 \mathrm{~nm}$ & 0.0537 & LUMO $\leftarrow$ HOMO-3 $(78 \%)$ \\
& & LUMO $+3 \leftarrow$ HOMO-3 $(12 \%)$ \\
\hline
\end{tabular}

compound exhibits an absorption maximum at $280 \mathrm{~nm}$ and a shoulder around $320 \mathrm{~nm}$ (Fig. 4a). The absorption maximum at $280 \mathrm{~nm}$ is typical of benzimidazole (Hirayama, 1967), and the $320 \mathrm{~nm}$ shoulder is typical of benzimidazole derivatives (Mosquera et al., 1996; Konoshima et al., 2012). The KubelkaMunk transformed spectrum of the solid sample is quite close to that of a structurally similar derivative reported earlier

(a)

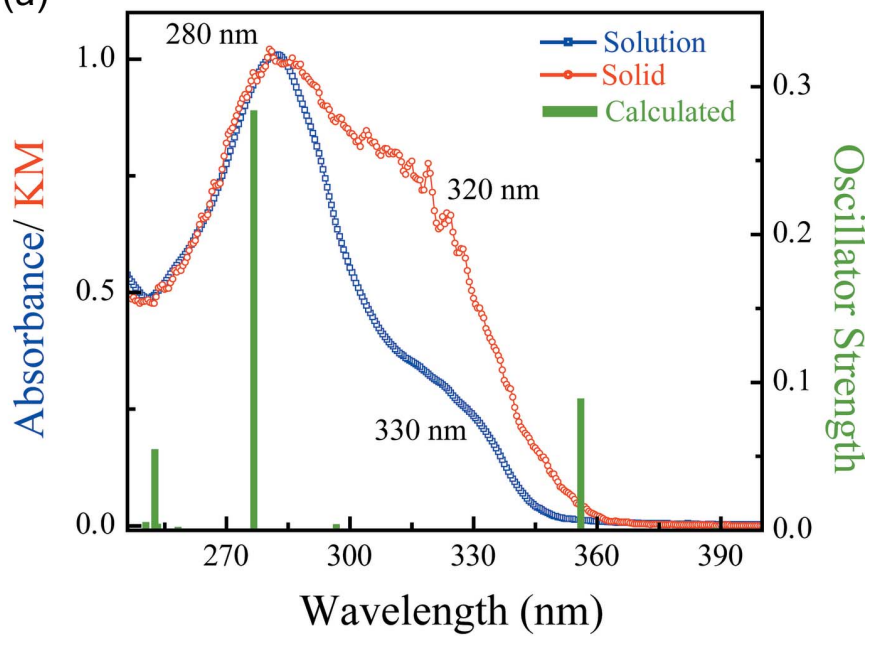

(b)

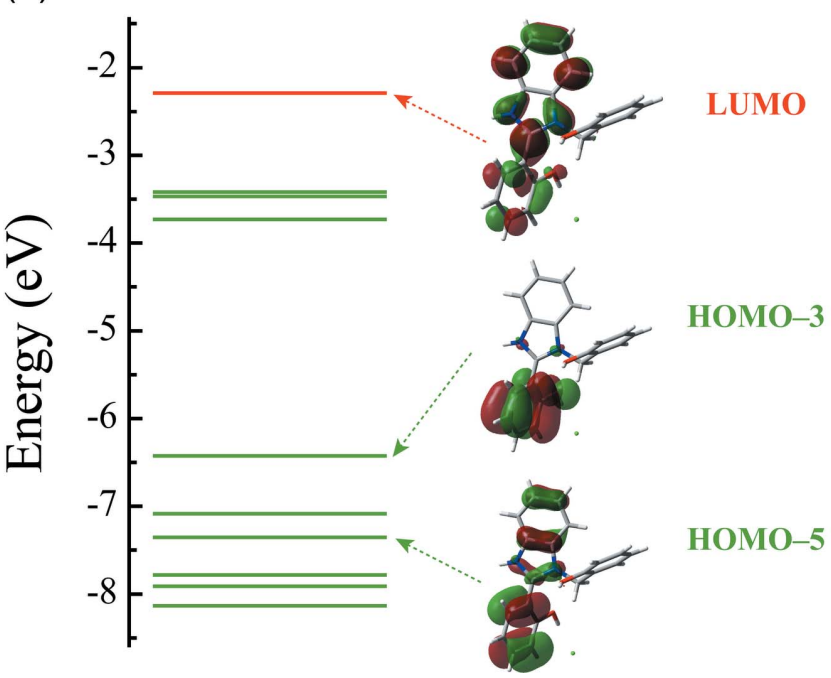

Figure 4

(a) Peak-normalized absorption spectrum of the compound in methanolic solution (blue), Kubelka-Munk (KM) transformed diffuse reflectance solid-state spectrum (red) and TD-DFT calculated transitions (green). (b) Molecular orbital energy levels and the relevant Kohn-Sham orbitals. 
(Shida et al., 2013). The positions and intensities of calculated transitions agree well with the experimental data (Fig. $4 a$, Table 3). The transition at $277 \mathrm{~nm}$ is found to have the $\pi-\pi^{*}$ nature. The associated molecular orbitals (HOMO-5 and LUMO) are spread over benzimidazole and 2-phenyl group (Fig. 4b, Table 3). On the other hand, HOMO-3 is localized on 2-phenyl group, making the transitions at $356 \mathrm{~nm}$ partially charge-transfer in nature.

\section{Database survey}

A survey of Cambridge Structure Database (CSD version 5.36, November 2016) (Groom et al., 2016) for molecules with the 2-[1-(2-hydroxybenzyl)-1H-benzo[d]imidazol-2-yl]phenol skeleton gave 18 hits. All of them are neutral molecules. Among them are an $o$-methylated derivative of the title compound (VIRZEC; Tarte et al., 2007), an o-ethoxy derivative (ZARFEF; Ha), $o$-methoxy derivatives (VOQVAZ and VOQRUP; Al-Douh et al., 2009a and Ha, 2012, respectively). Halide derivatives (CIQQOJ, NEGRIB) have also been reported (Fang et al., 2007; Yang et al., 2006). A search for protonated molecules containing the 1-benzyl-2-phenyl- $1 H$ benzo[ $d]$ imidazol-3-ium skeleton gave 11 hits, three of which being closely related to this work are reported in the same article (EBOHOU, EBOHUA and EBOJAI; Wang et al., 2011).

\section{Synthesis and crystallization}

Salicylaldehyde (SD Fine Chemicals, Mumbai, India), ophenylenediamine (Sigma- Aldrich, USA) and trimethylsilyl chloride (Sigma-Aldrich, USA) were used as received. The title compound was synthesized by the reaction of $o$-phenylenediamine $(1 \mathrm{~g})$ with salicylaldehyde (1:2 mole ratio) in double distilled water at $363 \mathrm{~K}$ using trimethylsilyl chloride as catalyst (1:1 molar ratio with respect to $o$-phenylenediamine) for 8-10 h (Wan et al., 2009). The reaction mixture was cooled to room temperature, and the white precipitate was filtered off, washed with water, dried by pressing against filter paper and allowed to dry at ambient conditions over a few days. Unexpectedly, the product turned out to be a salt, not a neutral compound, as prescribed by the literature synthetic procedure. It was crystallized from a solution in acetonitrile/ methanol mixture (15:85) in a refrigerator and then at room temperature. The resulting plate-shaped crystals were used for single crystal XRD measurements. Even after repeated attempts with crude and recrystallized samples, a clean ${ }^{1} \mathrm{H}$ NMR spectrum, which is an indication of rotamerism in solution, was not obtained. For the spectroscopic study, the parent solvent was decanted and then the crystals were washed with diethyl ether and finally air dried.

\section{Refinement}

Crystal data, data collection and structure refinement details are summarized in Table 4. All $\mathrm{H}$ atoms were positioned geometrically $(\mathrm{O}-\mathrm{H}=0.84, \mathrm{~N}-\mathrm{H}=0.88, \mathrm{C}-\mathrm{H}=0.95-$
Table 4

Experimental details.

\begin{tabular}{|c|c|}
\hline \multicolumn{2}{|l|}{ Crystal data } \\
\hline Chemical formula & $\mathrm{C}_{20} \mathrm{H}_{17} \mathrm{~N}_{2} \mathrm{O}_{2}^{+} \cdot \mathrm{Cl}^{-}$ \\
\hline$M_{\mathrm{r}}$ & 352.80 \\
\hline Crystal system, space group & Triclinic, $P \overline{1}$ \\
\hline Temperature $(\mathrm{K})$ & 150 \\
\hline$a, b, c(\AA)$ & $9.8002(4), 10.6791(5), 10.6986(4)$ \\
\hline$\alpha, \beta, \gamma\left({ }^{\circ}\right)$ & $\begin{array}{l}111.364(4), 102.346(3) \\
111.311(4)\end{array}$ \\
\hline$V\left(\AA^{3}\right)$ & $890.75(7)$ \\
\hline$Z$ & 2 \\
\hline Radiation type & Mo $K \alpha$ \\
\hline$\mu\left(\mathrm{mm}^{-1}\right)$ & 0.23 \\
\hline Crystal size $(\mathrm{mm})$ & $0.19 \times 0.18 \times 0.12$ \\
\hline
\end{tabular}

Data collection

Diffractometer

Absorption correction

$T_{\min }, T_{\max }$

No. of measured, independent and observed $[I>2 \sigma(I)]$ reflections

$R_{\text {int }}$

$(\sin \theta / \lambda)_{\max }\left(\AA^{-1}\right)$

Rigaku Saturn 724

Multi-scan (CrysAlis PRO; Rigaku Oxford Diffraction, 2015)

$0.657,1.000$

$8813,3114,2654$

0.033

0.595

Refinement

$R\left[F^{2}>2 \sigma\left(F^{2}\right)\right], w R\left(F^{2}\right), S$

No. of reflections

No. of parameters

$\mathrm{H}$-atom treatment

$\Delta \rho_{\max }, \Delta \rho_{\min }\left(\mathrm{e} \AA^{-3}\right)$

$0.039,0.105,1.04$

3114

228

$\mathrm{H}$-atom parameters constrained $0.25,-0.21$

Computer programs: CrysAlis PRO (Rigaku Oxford Diffraction, 2015), SHELXT (Sheldrick, 2015a), SHELXL2014 (Sheldrick, 2015b) and OLEX2 (Dolomanov et al., 2009).

$0.99 \AA$ ) and refined using a riding model with $U_{\text {iso }}(\mathrm{H})=$ $1.2 U_{\mathrm{eq}}(\mathrm{C}, \mathrm{N})\left[1.5 U_{\mathrm{eq}}(\mathrm{O})\right] . \mathrm{OH}$ groups were allowed to rotate about the $\mathrm{C}$-bonds.

\section{Acknowledgements}

TK thanks the CSIR and IRCC, IIT Bombay for a research fellowship. Department of Chemistry, IIT Bombay is acknowledged for the high-performance computing facility and single-crystal X-ray facility. The authors thank Archit Sanadhya, Harit Kumar and Pooja S. Varak for their efforts in synthesizing the title compound and also thank Shefali Vaidya for her help in drawing the packing diagram.

\section{Funding information}

Funding for this research was provided by: Science and Engineering Research Board (grant to Anindya Datta).

\section{References}

Al-Douh, M. H., Osman, H., Hamid, S. A., Kia, R. \& Fun, H.-K. (2009a). Acta Cryst. E65, o925-o926.

Al-Douh, M. H., Osman, H., Hamid, S. A., Kia, R. \& Fun, H.-K. (2009b). Acta Cryst. E65, o913-o914.

Benelhadj, K., Massue, J., Retailleau, P., Ulrich, G. \& Ziessel, R. (2013). Org. Lett. 15, 2918-2921.

Carvalho, L. C. R., Fernandes, E. \& Marques, M. M. B. (2011). Chem. Eur. J. 17, 12544-12555.

Dolomanov, O. V., Bourhis, L. J., Gildea, R. J., Howard, J. A. K. \& Puschmann, H. (2009). J. Appl. Cryst. 42, 339-341. 
Douhal, A., Amat-Guerri, F., Lillo, M. P. \& Acua, A. U. (1994). J. Photochem. Photobiol. Chem. 78, 127-138.

Fang, X.-N., Liu, L.-M., Huang, G.-S. \& Hu, P. (2007). Acta Cryst. E63, o4881.

Frisch, M. J., et al. (2009). GAUSSIAN09. Gaussian Inc., Wallingford, CT, USA.

Groom, C. R., Bruno, I. J., Lightfoot, M. P. \& Ward, S. C. (2016). Acta Cryst. B72, 171-179.

Ha, K. (2012). Acta Cryst. E68, o1398.

Hirayama, K. (1967). In Handbook of Ultraviolet and Visible Absorption Spectra of Organic Compounds. New York: Plenum Press Data Division.

Keri, R. S., Hiremathad, A., Budagumpi, S. \& Nagaraja, B. M. (2015). Chem. Biol. Drug Des. 86, 19-65.

Konoshima, H., Nagao, S., Kiyota, I., Amimoto, K., Yamamoto, N., Sekine, M., Nakata, M., Furukawa, K. \& Sekiya, H. (2012). Phys. Chem. Chem. Phys. 14, 16448-16457.

Kubelka, P. \& Munk, F. (1931). Z. Tech. Phys. 11A, 593-601.

Mosquera, M., Penedo, J. C., Ríos Rodríguez, M. C. \& RodríguezPrieto, F. (1996). J. Phys. Chem. 100, 5398-5407.

Rigaku Oxford Diffraction (2015). CrysAlis PRO. Rigaku Oxford Diffraction Ltd, Abingdon, England.
Ríos Vázquez, S., Ríos Rodríguez, M. C., Mosquera, M. \& RodríguezPrieto, F. (2008). J. Phys. Chem. A, 112, 376-387.

Sheldrick, G. M. (2015a). Acta Cryst. A71, 3-8.

Sheldrick, G. M. (2015b). Acta Cryst. C71, 3-8.

Shida, T., Mutai, T. \& Araki, K. (2013). CrystEngComm, 15, 10179 10182.

Soman, R., Sujatha, S. \& Arunkumar, C. (2014). J. Fluor. Chem. 163, $16-22$.

Spackman, M. A. \& Jayatilaka, D. (2009). CrystEngComm, 11, 19-32.

Sun, P., Jian, F. \& Tian, Y. (2011). J. Chem. Crystallogr. 41, 10491052.

Tarte, N. H., Cho, H. Y. \& Woo, S. I. (2007). Macromolecules, 40, 8162-8167.

Wan, J.-P., Gan, S.-F., Wu, J.-M. \& Pan, Y. (2009). Green Chem. 11, $1633-1637$.

Wang, Y.-T., Tang, G.-M., He, C., Yan, S.-C., Hao, Q.-C., Chen, L., Long, X.-F., Li, T.-D. \& Ng, S. W. (2011). CrystEngComm, 13, 63656368.

Yang, X., Jones, R. A., Lai, R. J., Waheed, A., Oye, M. M. \& Holmes, A. L. (2006). Polyhedron, 25, 881-887.

Zhao, D., Hu, J., Wu, N., Huang, X., Qin, X., Lan, J. \& You, J. (2011). Org. Lett. 13, 6516-6519. 


\section{supporting information}

Acta Cryst. (2017). E73, 1143-1147 [https://doi.org/10.1107/S205698901700977X]

\section{Crystal structure and UV spectra of a 1,2-disubstituted benzimidazolium chloride}

Tuhin Khan, Navneet Mishra, Darshan S. Mhatre and Anindya Datta

Computing details

Data collection: CrysAlis PRO (Rigaku Oxford Diffraction, 2015); cell refinement: CrysAlis PRO (Rigaku Oxford Diffraction, 2015); data reduction: CrysAlis PRO (Rigaku Oxford Diffraction, 2015); program(s) used to solve structure: SHELXT (Sheldrick, 2015a); program(s) used to refine structure: SHELXL2014 (Sheldrick, 2015b); molecular graphics: OLEX2 (Dolomanov et al., 2009); software used to prepare material for publication: OLEX2 (Dolomanov et al., 2009).

1-(2-Hydroxybenzyl)-2-(2-hydroxyphenyl)-1H-benzimidazol-3-ium chloride

\section{Crystal data}

$\mathrm{C}_{20} \mathrm{H}_{17} \mathrm{~N}_{2} \mathrm{O}_{2}^{+} \cdot \mathrm{Cl}^{-}$

$M_{r}=352.80$

Triclinic, $P \overline{1}$

$a=9.8002(4) \AA$

$b=10.6791(5) \AA$

$c=10.6986(4) \AA$

$\alpha=111.364(4)^{\circ}$

$\beta=102.346(3)^{\circ}$

$\gamma=111.311(4)^{\circ}$

$V=890.75(7) \AA^{3}$

\section{Data collection}

Rigaku Saturn 724 diffractometer

Radiation source: fine-focus sealed X-ray tube, Enhance (Mo) X-ray Source

Graphite monochromator

$\omega$ scans

Absorption correction: multi-scan

(CrysAlis PRO; Rigaku Oxford Diffraction, 2015)

\section{Refinement}

Refinement on $F^{2}$

Least-squares matrix: full

$R\left[F^{2}>2 \sigma\left(F^{2}\right)\right]=0.039$

$w R\left(F^{2}\right)=0.105$

$S=1.04$

3114 reflections

228 parameters

0 restraints
$Z=2$

$F(000)=368$

$D_{\mathrm{x}}=1.315 \mathrm{Mg} \mathrm{m}^{-3}$

Mo $K \alpha$ radiation, $\lambda=0.71073 \AA$

Cell parameters from 5921 reflections

$\theta=2.3-31.1^{\circ}$

$\mu=0.23 \mathrm{~mm}^{-1}$

$T=150 \mathrm{~K}$

Plate, colourless

$0.19 \times 0.18 \times 0.12 \mathrm{~mm}$

$T_{\min }=0.657, T_{\max }=1.000$

8813 measured reflections

3114 independent reflections

2654 reflections with $I>2 \sigma(I)$

$R_{\text {int }}=0.033$

$\theta_{\max }=25.0^{\circ}, \theta_{\min }=2.3^{\circ}$

$h=-11 \rightarrow 10$

$k=-12 \rightarrow 12$

$l=-11 \rightarrow 12$

Hydrogen site location: inferred from neighbouring sites

$\mathrm{H}$-atom parameters constrained

$w=1 /\left[\sigma^{2}\left(F_{\mathrm{o}}^{2}\right)+(0.0483 P)^{2}+0.2976 P\right]$

where $P=\left(F_{\mathrm{o}}^{2}+2 F_{\mathrm{c}}^{2}\right) / 3$

$(\Delta / \sigma)_{\max }=0.001$

$\Delta \rho_{\max }=0.25 \mathrm{e} \AA^{-3}$

$\Delta \rho_{\min }=-0.21$ e $\AA^{-3}$ 


\section{Special details}

Geometry. All esds (except the esd in the dihedral angle between two 1.s. planes) are estimated using the full covariance matrix. The cell esds are taken into account individually in the estimation of esds in distances, angles and torsion angles; correlations between esds in cell parameters are only used when they are defined by crystal symmetry. An approximate (isotropic) treatment of cell esds is used for estimating esds involving l.s. planes.

Refinement. 1. Fixed Uiso At 1.2 times of: All $\mathrm{C}(\mathrm{H})$ groups, All $\mathrm{C}(\mathrm{H}, \mathrm{H})$ groups, All $\mathrm{N}(\mathrm{H})$ groups At 1.5 times of: All $\mathrm{O}(\mathrm{H})$ groups 2.a Secondary $\mathrm{CH} 2$ refined with riding coordinates: $\mathrm{C} 7(\mathrm{H} 7 \mathrm{~A}, \mathrm{H} 7 \mathrm{~B}) 2 . \mathrm{b}$ Aromatic/amide $\mathrm{H}$ refined with riding coordinates: N2(H2A), C2(H2B), C3(H3), C4(H4), C5(H5), C9(H9), C10(H10), C11(H11), C12(H12), C16(H16), $\mathrm{C} 17(\mathrm{H} 17), \mathrm{C} 18(\mathrm{H} 18), \mathrm{C} 19(\mathrm{H} 19)$ 2.c Idealised tetrahedral $\mathrm{OH}$ refined as rotating group: O1(H1), O2(H2)

Fractional atomic coordinates and isotropic or equivalent isotropic displacement parameters $\left(\AA^{2}\right)$

\begin{tabular}{|c|c|c|c|c|}
\hline & $x$ & $y$ & $z$ & $U_{\text {iso }} * / U_{\text {eq }}$ \\
\hline $\mathrm{Cl1}$ & $-0.06343(6)$ & $0.67366(6)$ & $0.86942(5)$ & $0.04152(17)$ \\
\hline $\mathrm{O} 1$ & $0.57838(18)$ & $0.45573(18)$ & $0.75661(16)$ & $0.0457(4)$ \\
\hline $\mathrm{H} 1$ & 0.6774 & 0.5046 & 0.7816 & $0.069^{*}$ \\
\hline $\mathrm{O} 2$ & $0.07420(16)$ & $0.49890(14)$ & $0.68652(15)$ & $0.0381(3)$ \\
\hline $\mathrm{H} 2$ & 0.0365 & 0.5481 & 0.7349 & $0.057^{*}$ \\
\hline N1 & $0.23361(18)$ & $0.29524(16)$ & $0.60618(15)$ & $0.0302(3)$ \\
\hline $\mathrm{N} 2$ & $0.18508(19)$ & $0.27540(17)$ & $0.38883(16)$ & $0.0337(4)$ \\
\hline $\mathrm{H} 2 \mathrm{~A}$ & 0.1727 & 0.2994 & 0.3183 & $0.040^{*}$ \\
\hline $\mathrm{C} 7$ & $0.2833(2)$ & $0.3651(2)$ & $0.76670(19)$ & $0.0325(4)$ \\
\hline $\mathrm{H} 7 \mathrm{~A}$ & 0.1877 & 0.3293 & 0.7888 & $0.039^{*}$ \\
\hline H7B & 0.3331 & 0.4783 & 0.8101 & $0.039^{*}$ \\
\hline $\mathrm{C} 1$ & $0.5475(2)$ & $0.3725(2)$ & $0.8279(2)$ & $0.0355(4)$ \\
\hline $\mathrm{C} 2$ & $0.6572(3)$ & $0.3358(2)$ & $0.8901(2)$ & $0.0428(5)$ \\
\hline $\mathrm{H} 2 \mathrm{~B}$ & 0.7577 & 0.3671 & 0.8826 & $0.051^{*}$ \\
\hline $\mathrm{C} 3$ & $0.6179(3)$ & $0.2535(2)$ & $0.9626(2)$ & $0.0467(5)$ \\
\hline $\mathrm{H} 3$ & 0.6913 & 0.2262 & 1.0033 & $0.056^{*}$ \\
\hline $\mathrm{C} 4$ & $0.4745(3)$ & $0.2105(2)$ & $0.9770(2)$ & $0.0467(5)$ \\
\hline $\mathrm{H} 4$ & 0.4506 & 0.1571 & 1.0303 & $0.056^{*}$ \\
\hline $\mathrm{C} 5$ & $0.3645(3)$ & $0.2453(2)$ & $0.9129(2)$ & $0.0400(5)$ \\
\hline H5 & 0.2647 & 0.2148 & 0.9217 & $0.048^{*}$ \\
\hline C6 & $0.3999(2)$ & $0.3248(2)$ & $0.83576(18)$ & $0.0325(4)$ \\
\hline $\mathrm{C} 8$ & $0.1953(2)$ & $0.1454(2)$ & $0.50925(19)$ & $0.0321(4)$ \\
\hline C9 & $0.1862(2)$ & $0.0217(2)$ & $0.5291(2)$ & $0.0380(5)$ \\
\hline H9 & 0.2058 & 0.0277 & 0.6225 & $0.046^{*}$ \\
\hline $\mathrm{C} 10$ & $0.1472(3)$ & $-0.1105(2)$ & $0.4064(2)$ & $0.0428(5)$ \\
\hline H10 & 0.1417 & -0.1968 & 0.4163 & $0.051^{*}$ \\
\hline C11 & $0.1156(3)$ & $-0.1214(2)$ & $0.2682(2)$ & $0.0422(5)$ \\
\hline H11 & 0.0886 & -0.2150 & 0.1867 & $0.051^{*}$ \\
\hline $\mathrm{C} 12$ & $0.1225(2)$ & $-0.0002(2)$ & $0.2470(2)$ & $0.0384(5)$ \\
\hline H12 & 0.0995 & -0.0078 & 0.1528 & $0.046^{*}$ \\
\hline $\mathrm{C} 13$ & $0.1650(2)$ & $0.1346(2)$ & $0.3711(2)$ & $0.0333(4)$ \\
\hline $\mathrm{C} 14$ & $0.2263(2)$ & $0.3700(2)$ & $0.52966(19)$ & $0.0309(4)$ \\
\hline $\mathrm{C} 15$ & $0.2661(2)$ & $0.5309(2)$ & $0.58492(19)$ & $0.0319(4)$ \\
\hline $\mathrm{C} 16$ & $0.3810(2)$ & $0.6224(2)$ & $0.5524(2)$ & $0.0385(5)$ \\
\hline H16 & 0.4303 & 0.5785 & 0.4957 & $0.046^{*}$ \\
\hline
\end{tabular}




$\begin{array}{lllll}\text { C17 } & 0.4234(3) & 0.7744(2) & 0.6010(2) & 0.0428(5) \\ \text { H17 } & 0.5025 & 0.8362 & 0.5796 & 0.051^{*} \\ \text { C18 } & 0.3493(3) & 0.8366(2) & 0.6819(2) & 0.0402(5) \\ \text { H18 } & 0.3796 & 0.9423 & 0.7175 & 0.048^{*} \\ \text { C19 } & 0.2325(2) & 0.7481(2) & 0.7116(2) & 0.0356(4) \\ \text { H19 } & 0.1812 & 0.7922 & 0.7651 & 0.043^{*} \\ \text { C20 } & 0.1895(2) & 0.5940(2) & 0.66327(19) & 0.0314(4)\end{array}$

Atomic displacement parameters $\left(\AA^{2}\right)$

\begin{tabular}{lllllll}
\hline & $U^{11}$ & $U^{22}$ & $U^{33}$ & $U^{12}$ & $U^{13}$ & $U^{23}$ \\
\hline C11 & $0.0437(3)$ & $0.0520(3)$ & $0.0327(3)$ & $0.0275(3)$ & $0.0155(2)$ & $0.0196(2)$ \\
O1 & $0.0415(9)$ & $0.0639(10)$ & $0.0441(8)$ & $0.0290(8)$ & $0.0202(7)$ & $0.0329(8)$ \\
O2 & $0.0398(8)$ & $0.0354(7)$ & $0.0450(8)$ & $0.0232(7)$ & $0.0205(7)$ & $0.0183(6)$ \\
N1 & $0.0339(9)$ & $0.0275(8)$ & $0.0257(8)$ & $0.0183(7)$ & $0.0072(7)$ & $0.0089(6)$ \\
N2 & $0.0394(10)$ & $0.0352(8)$ & $0.0272(8)$ & $0.0214(8)$ & $0.0109(7)$ & $0.0139(7)$ \\
C7 & $0.0370(11)$ & $0.0326(10)$ & $0.0250(9)$ & $0.0199(9)$ & $0.0095(8)$ & $0.0099(8)$ \\
C1 & $0.0420(12)$ & $0.0379(10)$ & $0.0254(9)$ & $0.0232(9)$ & $0.0114(9)$ & $0.0117(8)$ \\
C2 & $0.0449(13)$ & $0.0488(12)$ & $0.0330(11)$ & $0.0305(11)$ & $0.0106(10)$ & $0.0131(9)$ \\
C3 & $0.0574(15)$ & $0.0436(12)$ & $0.0340(11)$ & $0.0329(11)$ & $0.0055(10)$ & $0.0127(10)$ \\
C4 & $0.0639(16)$ & $0.0346(11)$ & $0.0321(11)$ & $0.0226(11)$ & $0.0068(11)$ & $0.0154(9)$ \\
C5 & $0.0452(13)$ & $0.0335(10)$ & $0.0313(10)$ & $0.0161(9)$ & $0.0094(9)$ & $0.0126(9)$ \\
C6 & $0.0401(11)$ & $0.0295(9)$ & $0.0217(9)$ & $0.0192(9)$ & $0.0073(8)$ & $0.0070(8)$ \\
C8 & $0.0312(11)$ & $0.0289(9)$ & $0.0292(10)$ & $0.0172(8)$ & $0.0066(8)$ & $0.0079(8)$ \\
C9 & $0.0400(12)$ & $0.0343(10)$ & $0.0343(10)$ & $0.0206(9)$ & $0.0074(9)$ & $0.0131(9)$ \\
C10 & $0.0435(13)$ & $0.0312(10)$ & $0.0451(12)$ & $0.0222(10)$ & $0.0078(10)$ & $0.0117(9)$ \\
C11 & $0.0391(12)$ & $0.0329(10)$ & $0.0366(11)$ & $0.0190(9)$ & $0.0069(9)$ & $0.0027(9)$ \\
C12 & $0.0364(12)$ & $0.0385(11)$ & $0.0278(10)$ & $0.0192(9)$ & $0.0064(9)$ & $0.0067(8)$ \\
C13 & $0.0291(10)$ & $0.0337(10)$ & $0.0317(10)$ & $0.0178(9)$ & $0.0079(8)$ & $0.0106(8)$ \\
C14 & $0.0293(10)$ & $0.0332(10)$ & $0.0291(9)$ & $0.0187(8)$ & $0.0084(8)$ & $0.0123(8)$ \\
C15 & $0.0339(11)$ & $0.0327(10)$ & $0.0278(9)$ & $0.0195(9)$ & $0.0072(8)$ & $0.0130(8)$ \\
C16 & $0.0437(12)$ & $0.0436(11)$ & $0.0380(11)$ & $0.0276(10)$ & $0.0184(10)$ & $0.0216(9)$ \\
C17 & $0.0440(13)$ & $0.0415(11)$ & $0.0499(12)$ & $0.0220(10)$ & $0.0189(11)$ & $0.0274(10)$ \\
C18 & $0.0467(13)$ & $0.0311(10)$ & $0.0404(11)$ & $0.0203(10)$ & $0.0094(10)$ & $0.0181(9)$ \\
C19 & $0.0418(12)$ & $0.0365(10)$ & $0.0326(10)$ & $0.0262(10)$ & $0.0113(9)$ & $0.0157(9)$ \\
C20 & $0.0313(10)$ & $0.0340(10)$ & $0.0278(9)$ & $0.0178(9)$ & $0.0073(8)$ & $0.0145(8)$ \\
& & & & & & \\
\hline & & & & & & \\
\end{tabular}

Geometric parameters $\left(\AA,{ }^{\circ}\right)$

\begin{tabular}{llll}
\hline $\mathrm{O} 1-\mathrm{H} 1$ & 0.8400 & $\mathrm{C} 5-\mathrm{C} 6$ & $1.393(3)$ \\
$\mathrm{O} 1-\mathrm{C} 1$ & $1.365(2)$ & $\mathrm{C} 8-\mathrm{C} 9$ & $1.388(3)$ \\
$\mathrm{O} 2-\mathrm{H} 2$ & 0.8400 & $\mathrm{C} 8-\mathrm{C} 13$ & $1.396(3)$ \\
$\mathrm{O} 2-\mathrm{C} 20$ & $1.356(2)$ & $\mathrm{C} 9-\mathrm{H} 9$ & 0.9500 \\
$\mathrm{~N} 1-\mathrm{C} 7$ & $1.479(2)$ & $\mathrm{C} 9-\mathrm{C} 10$ & $1.380(3)$ \\
$\mathrm{N} 1-\mathrm{C} 8$ & $1.402(2)$ & $\mathrm{C} 10-\mathrm{H} 10$ & 0.9500 \\
$\mathrm{~N} 1-\mathrm{C} 14$ & $1.344(2)$ & $\mathrm{C} 10-\mathrm{C} 11$ & $1.396(3)$ \\
$\mathrm{N} 2-\mathrm{H} 2 \mathrm{~A}$ & 0.8800 & $\mathrm{C} 11-\mathrm{H} 11$ & 0.9500 \\
$\mathrm{~N} 2-\mathrm{C} 13$ & $1.378(2)$ & $\mathrm{C} 11-\mathrm{C} 12$ & $1.374(3)$
\end{tabular}




\begin{tabular}{|c|c|c|c|}
\hline $\mathrm{N} 2-\mathrm{C} 14$ & $1.337(2)$ & $\mathrm{C} 12-\mathrm{H} 12$ & 0.9500 \\
\hline $\mathrm{C} 7-\mathrm{H} 7 \mathrm{~A}$ & 0.9900 & $\mathrm{C} 12-\mathrm{C} 13$ & $1.394(2)$ \\
\hline C7-H7B & 0.9900 & $\mathrm{C} 14-\mathrm{C} 15$ & $1.458(2)$ \\
\hline $\mathrm{C} 7-\mathrm{C} 6$ & $1.505(2)$ & $\mathrm{C} 15-\mathrm{C} 16$ & $1.400(3)$ \\
\hline $\mathrm{C} 1-\mathrm{C} 2$ & $1.393(3)$ & $\mathrm{C} 15-\mathrm{C} 20$ & $1.396(3)$ \\
\hline $\mathrm{C} 1-\mathrm{C} 6$ & $1.381(3)$ & $\mathrm{C} 16-\mathrm{H} 16$ & 0.9500 \\
\hline $\mathrm{C} 2-\mathrm{H} 2 \mathrm{~B}$ & 0.9500 & $\mathrm{C} 16-\mathrm{C} 17$ & $1.371(3)$ \\
\hline $\mathrm{C} 2-\mathrm{C} 3$ & $1.380(3)$ & $\mathrm{C} 17-\mathrm{H} 17$ & 0.9500 \\
\hline $\mathrm{C} 3-\mathrm{H} 3$ & 0.9500 & $\mathrm{C} 17-\mathrm{C} 18$ & $1.385(3)$ \\
\hline $\mathrm{C} 3-\mathrm{C} 4$ & $1.373(3)$ & $\mathrm{C} 18-\mathrm{H} 18$ & 0.9500 \\
\hline $\mathrm{C} 4-\mathrm{H} 4$ & 0.9500 & $\mathrm{C} 18-\mathrm{C} 19$ & $1.375(3)$ \\
\hline $\mathrm{C} 4-\mathrm{C} 5$ & $1.391(3)$ & $\mathrm{C} 19-\mathrm{H} 19$ & 0.9500 \\
\hline $\mathrm{C} 5-\mathrm{H} 5$ & 0.9500 & $\mathrm{C} 19-\mathrm{C} 20$ & $1.391(3)$ \\
\hline $\mathrm{C} 1-\mathrm{O} 1-\mathrm{H} 1$ & 109.5 & $\mathrm{C} 10-\mathrm{C} 9-\mathrm{C} 8$ & $116.71(18)$ \\
\hline $\mathrm{C} 20-\mathrm{O} 2-\mathrm{H} 2$ & 109.5 & $\mathrm{C} 10-\mathrm{C} 9-\mathrm{H} 9$ & 121.6 \\
\hline $\mathrm{C} 8-\mathrm{N} 1-\mathrm{C} 7$ & $126.87(14)$ & $\mathrm{C} 9-\mathrm{C} 10-\mathrm{H} 10$ & 118.9 \\
\hline $\mathrm{C} 14-\mathrm{N} 1-\mathrm{C} 7$ & $124.73(14)$ & $\mathrm{C} 9-\mathrm{C} 10-\mathrm{C} 11$ & $122.14(18)$ \\
\hline $\mathrm{C} 14-\mathrm{N} 1-\mathrm{C} 8$ & $108.30(14)$ & $\mathrm{C} 11-\mathrm{C} 10-\mathrm{H} 10$ & 118.9 \\
\hline $\mathrm{C} 13-\mathrm{N} 2-\mathrm{H} 2 \mathrm{~A}$ & 125.2 & $\mathrm{C} 10-\mathrm{C} 11-\mathrm{H} 11$ & 119.2 \\
\hline $\mathrm{C} 14-\mathrm{N} 2-\mathrm{H} 2 \mathrm{~A}$ & 125.2 & $\mathrm{C} 12-\mathrm{C} 11-\mathrm{C} 10$ & $121.63(17)$ \\
\hline $\mathrm{C} 14-\mathrm{N} 2-\mathrm{C} 13$ & $109.54(15)$ & $\mathrm{C} 12-\mathrm{C} 11-\mathrm{H} 11$ & 119.2 \\
\hline $\mathrm{N} 1-\mathrm{C} 7-\mathrm{H} 7 \mathrm{~A}$ & 109.2 & $\mathrm{C} 11-\mathrm{C} 12-\mathrm{H} 12$ & 121.8 \\
\hline $\mathrm{N} 1-\mathrm{C} 7-\mathrm{H} 7 \mathrm{~B}$ & 109.2 & $\mathrm{C} 11-\mathrm{C} 12-\mathrm{C} 13$ & $116.42(18)$ \\
\hline $\mathrm{N} 1-\mathrm{C} 7-\mathrm{C} 6$ & $112.25(14)$ & $\mathrm{C} 13-\mathrm{C} 12-\mathrm{H} 12$ & 121.8 \\
\hline $\mathrm{H} 7 \mathrm{~A}-\mathrm{C} 7-\mathrm{H} 7 \mathrm{~B}$ & 107.9 & $\mathrm{~N} 2-\mathrm{C} 13-\mathrm{C} 8$ & $106.63(15)$ \\
\hline $\mathrm{C} 6-\mathrm{C} 7-\mathrm{H} 7 \mathrm{~A}$ & 109.2 & $\mathrm{~N} 2-\mathrm{C} 13-\mathrm{C} 12$ & $131.29(17)$ \\
\hline $\mathrm{C} 6-\mathrm{C} 7-\mathrm{H} 7 \mathrm{~B}$ & 109.2 & $\mathrm{C} 12-\mathrm{C} 13-\mathrm{C} 8$ & $122.08(17)$ \\
\hline $\mathrm{O} 1-\mathrm{C} 1-\mathrm{C} 2$ & $122.67(19)$ & $\mathrm{N} 1-\mathrm{C} 14-\mathrm{C} 15$ & $128.09(16)$ \\
\hline $\mathrm{O} 1-\mathrm{C} 1-\mathrm{C} 6$ & $116.49(16)$ & $\mathrm{N} 2-\mathrm{C} 14-\mathrm{N} 1$ & $109.17(15)$ \\
\hline $\mathrm{C} 6-\mathrm{C} 1-\mathrm{C} 2$ & $120.84(18)$ & $\mathrm{N} 2-\mathrm{C} 14-\mathrm{C} 15$ & $122.61(15)$ \\
\hline $\mathrm{C} 1-\mathrm{C} 2-\mathrm{H} 2 \mathrm{~B}$ & 120.4 & $\mathrm{C} 16-\mathrm{C} 15-\mathrm{C} 14$ & $118.21(16)$ \\
\hline $\mathrm{C} 3-\mathrm{C} 2-\mathrm{C} 1$ & $119.1(2)$ & $\mathrm{C} 20-\mathrm{C} 15-\mathrm{C} 14$ & $122.35(17)$ \\
\hline $\mathrm{C} 3-\mathrm{C} 2-\mathrm{H} 2 \mathrm{~B}$ & 120.4 & $\mathrm{C} 20-\mathrm{C} 15-\mathrm{C} 16$ & $119.39(17)$ \\
\hline $\mathrm{C} 2-\mathrm{C} 3-\mathrm{H} 3$ & 119.5 & $\mathrm{C} 15-\mathrm{C} 16-\mathrm{H} 16$ & 119.5 \\
\hline $\mathrm{C} 4-\mathrm{C} 3-\mathrm{C} 2$ & $121.02(19)$ & $\mathrm{C} 17-\mathrm{C} 16-\mathrm{C} 15$ & $120.94(18)$ \\
\hline $\mathrm{C} 4-\mathrm{C} 3-\mathrm{H} 3$ & 119.5 & $\mathrm{C} 17-\mathrm{C} 16-\mathrm{H} 16$ & 119.5 \\
\hline $\mathrm{C} 3-\mathrm{C} 4-\mathrm{H} 4$ & 120.2 & $\mathrm{C} 16-\mathrm{C} 17-\mathrm{H} 17$ & 120.5 \\
\hline $\mathrm{C} 3-\mathrm{C} 4-\mathrm{C} 5$ & $119.60(19)$ & $\mathrm{C} 16-\mathrm{C} 17-\mathrm{C} 18$ & $119.01(19)$ \\
\hline $\mathrm{C} 5-\mathrm{C} 4-\mathrm{H} 4$ & 120.2 & $\mathrm{C} 18-\mathrm{C} 17-\mathrm{H} 17$ & 120.5 \\
\hline $\mathrm{C} 4-\mathrm{C} 5-\mathrm{H} 5$ & 119.8 & $\mathrm{C} 17-\mathrm{C} 18-\mathrm{H} 18$ & 119.4 \\
\hline $\mathrm{C} 4-\mathrm{C} 5-\mathrm{C} 6$ & $120.3(2)$ & $\mathrm{C} 19-\mathrm{C} 18-\mathrm{C} 17$ & $121.23(18)$ \\
\hline $\mathrm{C} 6-\mathrm{C} 5-\mathrm{H} 5$ & 119.8 & $\mathrm{C} 19-\mathrm{C} 18-\mathrm{H} 18$ & 119.4 \\
\hline $\mathrm{C} 1-\mathrm{C} 6-\mathrm{C} 7$ & $119.68(16)$ & $\mathrm{C} 18-\mathrm{C} 19-\mathrm{H} 19$ & 120.0 \\
\hline $\mathrm{C} 1-\mathrm{C} 6-\mathrm{C} 5$ & $119.00(18)$ & $\mathrm{C} 18-\mathrm{C} 19-\mathrm{C} 20$ & $120.08(17)$ \\
\hline $\mathrm{C} 5-\mathrm{C} 6-\mathrm{C} 7$ & $121.27(18)$ & $\mathrm{C} 20-\mathrm{C} 19-\mathrm{H} 19$ & 120.0 \\
\hline $\mathrm{C} 9-\mathrm{C} 8-\mathrm{N} 1$ & $132.62(17)$ & $\mathrm{O} 2-\mathrm{C} 20-\mathrm{C} 15$ & $117.13(16)$ \\
\hline $\mathrm{C} 9-\mathrm{C} 8-\mathrm{C} 13$ & $121.01(16)$ & $\mathrm{O} 2-\mathrm{C} 20-\mathrm{C} 19$ & $123.57(16)$ \\
\hline
\end{tabular}




\begin{tabular}{|c|c|c|c|}
\hline $\mathrm{C} 13-\mathrm{C} 8-\mathrm{N} 1$ & $106.36(15)$ & $\mathrm{C} 19-\mathrm{C} 20-\mathrm{C} 15$ & $119.29(17)$ \\
\hline $\mathrm{C} 8-\mathrm{C} 9-\mathrm{H} 9$ & 121.6 & & \\
\hline $\mathrm{O} 1-\mathrm{C} 1-\mathrm{C} 2-\mathrm{C} 3$ & $178.87(18)$ & $\mathrm{C} 8-\mathrm{C} 9-\mathrm{C} 10-\mathrm{C} 11$ & $-1.1(3)$ \\
\hline $\mathrm{O} 1-\mathrm{C} 1-\mathrm{C} 6-\mathrm{C} 7$ & $0.3(2)$ & $\mathrm{C} 9-\mathrm{C} 8-\mathrm{C} 13-\mathrm{N} 2$ & $-179.52(17)$ \\
\hline $\mathrm{O} 1-\mathrm{C} 1-\mathrm{C} 6-\mathrm{C} 5$ & $-177.18(16)$ & $\mathrm{C} 9-\mathrm{C} 8-\mathrm{C} 13-\mathrm{C} 12$ & $0.8(3)$ \\
\hline $\mathrm{N} 1-\mathrm{C} 7-\mathrm{C} 6-\mathrm{C} 1$ & $63.1(2)$ & $\mathrm{C} 9-\mathrm{C} 10-\mathrm{C} 11-\mathrm{C} 12$ & $0.4(3)$ \\
\hline $\mathrm{N} 1-\mathrm{C} 7-\mathrm{C} 6-\mathrm{C} 5$ & $-119.49(18)$ & $\mathrm{C} 10-\mathrm{C} 11-\mathrm{C} 12-\mathrm{C} 13$ & $0.9(3)$ \\
\hline $\mathrm{N} 1-\mathrm{C} 8-\mathrm{C} 9-\mathrm{C} 10$ & $-178.6(2)$ & $\mathrm{C} 11-\mathrm{C} 12-\mathrm{C} 13-\mathrm{N} 2$ & $178.91(19)$ \\
\hline $\mathrm{N} 1-\mathrm{C} 8-\mathrm{C} 13-\mathrm{N} 2$ & $-0.2(2)$ & $\mathrm{C} 11-\mathrm{C} 12-\mathrm{C} 13-\mathrm{C} 8$ & $-1.5(3)$ \\
\hline $\mathrm{N} 1-\mathrm{C} 8-\mathrm{C} 13-\mathrm{C} 12$ & $-179.86(17)$ & $\mathrm{C} 13-\mathrm{N} 2-\mathrm{C} 14-\mathrm{N} 1$ & $-0.2(2)$ \\
\hline $\mathrm{N} 1-\mathrm{C} 14-\mathrm{C} 15-\mathrm{C} 16$ & $123.4(2)$ & $\mathrm{C} 13-\mathrm{N} 2-\mathrm{C} 14-\mathrm{C} 15$ & $175.93(16)$ \\
\hline $\mathrm{N} 1-\mathrm{C} 14-\mathrm{C} 15-\mathrm{C} 20$ & $-59.2(3)$ & $\mathrm{C} 13-\mathrm{C} 8-\mathrm{C} 9-\mathrm{C} 10$ & $0.5(3)$ \\
\hline $\mathrm{N} 2-\mathrm{C} 14-\mathrm{C} 15-\mathrm{C} 16$ & $-51.9(3)$ & $\mathrm{C} 14-\mathrm{N} 1-\mathrm{C} 7-\mathrm{C} 6$ & $-134.04(18)$ \\
\hline $\mathrm{N} 2-\mathrm{C} 14-\mathrm{C} 15-\mathrm{C} 20$ & $125.5(2)$ & $\mathrm{C} 14-\mathrm{N} 1-\mathrm{C} 8-\mathrm{C} 9$ & $179.3(2)$ \\
\hline $\mathrm{C} 7-\mathrm{N} 1-\mathrm{C} 8-\mathrm{C} 9$ & $2.7(3)$ & $\mathrm{C} 14-\mathrm{N} 1-\mathrm{C} 8-\mathrm{C} 13$ & $0.1(2)$ \\
\hline $\mathrm{C} 7-\mathrm{N} 1-\mathrm{C} 8-\mathrm{C} 13$ & $-176.58(17)$ & $\mathrm{C} 14-\mathrm{N} 2-\mathrm{C} 13-\mathrm{C} 8$ & $0.2(2)$ \\
\hline $\mathrm{C} 7-\mathrm{N} 1-\mathrm{C} 14-\mathrm{N} 2$ & $176.79(16)$ & $\mathrm{C} 14-\mathrm{N} 2-\mathrm{C} 13-\mathrm{C} 12$ & $179.9(2)$ \\
\hline $\mathrm{C} 7-\mathrm{N} 1-\mathrm{C} 14-\mathrm{C} 15$ & $1.0(3)$ & $\mathrm{C} 14-\mathrm{C} 15-\mathrm{C} 16-\mathrm{C} 17$ & $179.90(18)$ \\
\hline $\mathrm{C} 1-\mathrm{C} 2-\mathrm{C} 3-\mathrm{C} 4$ & $-1.4(3)$ & $\mathrm{C} 14-\mathrm{C} 15-\mathrm{C} 20-\mathrm{O} 2$ & $-0.1(3)$ \\
\hline $\mathrm{C} 2-\mathrm{C} 1-\mathrm{C} 6-\mathrm{C} 7$ & $-179.56(16)$ & $\mathrm{C} 14-\mathrm{C} 15-\mathrm{C} 20-\mathrm{C} 19$ & $-179.40(17)$ \\
\hline $\mathrm{C} 2-\mathrm{C} 1-\mathrm{C} 6-\mathrm{C} 5$ & $3.0(3)$ & $\mathrm{C} 15-\mathrm{C} 16-\mathrm{C} 17-\mathrm{C} 18$ & $-0.8(3)$ \\
\hline $\mathrm{C} 2-\mathrm{C} 3-\mathrm{C} 4-\mathrm{C} 5$ & $2.4(3)$ & $\mathrm{C} 16-\mathrm{C} 15-\mathrm{C} 20-\mathrm{O} 2$ & $177.20(16)$ \\
\hline $\mathrm{C} 3-\mathrm{C} 4-\mathrm{C} 5-\mathrm{C} 6$ & $-0.6(3)$ & $\mathrm{C} 16-\mathrm{C} 15-\mathrm{C} 20-\mathrm{C} 19$ & $-2.1(3)$ \\
\hline $\mathrm{C} 4-\mathrm{C} 5-\mathrm{C} 6-\mathrm{C} 7$ & $-179.43(17)$ & $\mathrm{C} 16-\mathrm{C} 17-\mathrm{C} 18-\mathrm{C} 19$ & $-1.2(3)$ \\
\hline $\mathrm{C} 4-\mathrm{C} 5-\mathrm{C} 6-\mathrm{C} 1$ & $-2.0(3)$ & $\mathrm{C} 17-\mathrm{C} 18-\mathrm{C} 19-\mathrm{C} 20$ & $1.6(3)$ \\
\hline $\mathrm{C} 6-\mathrm{C} 1-\mathrm{C} 2-\mathrm{C} 3$ & $-1.3(3)$ & $\mathrm{C} 18-\mathrm{C} 19-\mathrm{C} 20-\mathrm{O} 2$ & $-179.12(17)$ \\
\hline $\mathrm{C} 8-\mathrm{N} 1-\mathrm{C} 7-\mathrm{C} 6$ & $42.1(2)$ & $\mathrm{C} 18-\mathrm{C} 19-\mathrm{C} 20-\mathrm{C} 15$ & $0.1(3)$ \\
\hline $\mathrm{C} 8-\mathrm{N} 1-\mathrm{C} 14-\mathrm{N} 2$ & $0.1(2)$ & $\mathrm{C} 20-\mathrm{C} 15-\mathrm{C} 16-\mathrm{C} 17$ & $2.5(3)$ \\
\hline $\mathrm{C} 8-\mathrm{N} 1-\mathrm{C} 14-\mathrm{C} 15$ & $-175.76(18)$ & & \\
\hline
\end{tabular}

Hydrogen-bond geometry $\left(A,{ }^{\circ}\right)$

\begin{tabular}{lllll}
\hline$D-\mathrm{H} \cdots A$ & $D-\mathrm{H}$ & $\mathrm{H} \cdots A$ & $D \cdots A$ & $D-\mathrm{H} \cdots A$ \\
\hline $\mathrm{O} 1-\mathrm{H} 1 \cdots \mathrm{C} 11^{\mathrm{i}}$ & 0.84 & 2.24 & $3.066(2)$ & 169 \\
$\mathrm{O} 2-\mathrm{H} 2 \cdots \mathrm{C} 11$ & 0.84 & 2.23 & $3.071(1)$ & 177 \\
$\mathrm{~N} 2-\mathrm{H} 2 A \cdots \mathrm{C} 11^{\mathrm{ii}}$ & 0.88 & 2.23 & $3.084(2)$ & 162 \\
$\mathrm{C} 16-\mathrm{H} 16 \cdots \mathrm{O} 1^{\mathrm{iii}}$ & 0.95 & 2.57 & $3.253(2)$ & 129 \\
$\mathrm{C} 19-\mathrm{H} 19 \cdots \mathrm{Cl1}$ & 0.95 & 2.93 & $3.646(2)$ & 134 \\
$\mathrm{C} 7-\mathrm{H} 7 B \cdots \mathrm{Cg}(\mathrm{C} 1-\mathrm{C} 6)^{\mathrm{iv}}$ & 0.99 & 2.77 & $3.500(2)$ & 131 \\
\hline
\end{tabular}

Symmetry codes: (i) $x+1, y, z$; (ii) $-x,-y+1,-z+1$; (iii) $-x+1,-y+1,-z+1$; (iv) $-x+1,-y+1,-z+2$. 